\title{
THE POWER OF MUSIC
}

\author{
Dr. Seema sharma \\ Dept.of political science \\ Govt. M.L.B. Girls P.G. College, Indore
}

Since time immemorial, music has been embedded in life. It seeps into arts and culture, finds expression in language, and has great affect on lifestyle. The origin of music dates back to the earliest of civilization, thereby making it difficult to pinpoint the exact date whence it started. Robert Schumann, a German composer, noted, "Perhaps it is precisely the mystery of her origins which accounts for the charm of her beauty.

Whether vocal or instrumental, music can be viewed as a form of language or speech, possessing subtler shades of meaning than the spoken word and yet displaying much more emotive force. There seems to be something innate in human being that gives them the capacity to understand and respond to musical tones, rhythms, and pattern.

Nowadays, music has become so widespread all thanks to the latest innovations and technology that permit easy access and acquisition. Form the near- defunct cassette tapes, walkmans, and CD players to the more modern MP3 players and Apple`s iPod line, it has become possible for people to listen to any sort music simultaneously, conveniently, and privately.

Music has been known to have many positive effects on its listeners. In fact, a growing branch in medicine called Music Therapy is rapidly gaining popularity in recent years. This therapy claims to speed up the recovery process of patients, whether for physical therapy or post operative recuperation. Listening to relaxing or stimulating music has the capacity to accelerate the process of recovery for most patients, through this effect is largely dependent on the patients response to it - henceforth, this kind of therapy is not the primary means for recovery, but merely supplementary.

Aside from music's health benefits, music also enhances creativity. It has the possibility to be muses that allow people to get their creative juices flowing. Since music is administered to both the left and right side of the brains, it can help spark inspiration that might just be a proving to be a little elusive. To most Artists, listening to music can help them archive a certain creativity or ingenuity that they would be able to do simply on their own. Music also has an effect in a person`s task performance, since it can change moods and give more motivation to the listener. Studies show that music does not automatically increase positive attitude, for it will ultimately depend on the manner by which the music is played. It may, however, reduce the strain of an activity. As a rule, it is important that listener play music he is familiar and comfortable with, in a volume that is not too loud so as not to distract the listener. 


\section{INTERNATIONAL JOURNAL Of RESEARCH -GRANTHAALAYAH \\ A knowledge Repository}

Art

There are many other variables that contribute to the effects of music, but there is certainly in the fact that music has an effect on human, and therefore an effect on task performances. Generally, however, the effect of music has usually been more on the positive side than the negative. So pop on your earphones, have a listen, and Music therapy is increasingly used in hospitals to reduce the need for medication during childbirth, to decreases postoperative pain and complement the use of anesthesia during surgery. It is intrinsic to all cultures and has surprising benefits for not only learning language, improving memory and focusing attention, but also physical coordination and development.

Music can help reduce both the sensation and distress of both chronic pain and postoperative pain.

- Show music relaxes by slowing breathing and heartbeat.

\section{REDUCING BLOOD PRESSURE}

By playing recording of relaxing music every morning and evening, people with high blood pressure can train themselves to lower their blood pressure and keep it low.

When stroke patients in listened to music for a couple of hours each day, verbal memory and attention span improved significantly compared to patients who received no musical stimulation, or who listened only to story.

Music can help migraine and chronic headache sufferers reduce the intensity, frequency, and duration of the headaches.

Scientists explain that a particular type of music can create a positive and profound emotional experience, which leads to secretion of immune-boosting hormones. This helps contribute to a reduction in the factors responsible for illness. Listening to music or singing can also decrease levels of stress-related hormone cortical. Higher levels of cortical can lead to a decreased immune response. Using music therapy during childbirth decreased post-natal anxiety and pain, increases the satisfaction with childbirth and reduces the likelihood of postpartum depression.

\section{EFFECT OF MUSIC ON THE BRAIN}

\section{MUSIC ENHANCES INTELLIGENCE, LEARNING AND IQ}

Music has the power to enhance some kinds of higher brain function: Even children with attention deficit/hyperactivity disorder benefit in mathematics test from listening to music beforehand. Any music that is personally enjoyable has positive effects on cognition.

\section{MUSIC IMPROVES MEMORY PERFORMANCE}

The information being studied activates the left-brain while the music activates the right brain. Also, easy listening music or relaxing classics improves the duration and intensity of 


\section{INTERNATIONAL JOURNAL Of RESEARCH -GRANTHAALAYAH

concentration in all age groups and ability levels activities which engage both sides of the brain at the same time, such as playing an instrument or singing, cause the brain to be more capable of processing information. Certain types of music are a great "Keys" for recalling memories. Information learned while listening to a particular song can often be recalled simply by "playing" the song mentally. There is clear evidence, that children who take Music improve body movement and coordination.

Music reduces muscle tension and improves body movement and coordination. Music may play an important role in developing, maintain and restoring physical functioning in the rehabilitation of persons with movement disorders.

Music lessons develop a better memory compared with children who have no musical training. Choosing music that motivates you will make it easier to start moving, walking, dancing, or any other type of exercise-induced fatigue and fatigue symptoms caused by monotonous work. Many people like to listen to music while they work.

\section{RELAXING MUSIC INDUCES SLEEP}

Relaxing classical music is safe, cheap and easy way to beat insomnia. Many people who suffer from insomnia find that Bach music helps them. Relaxing music reduces sympathetic nervous system activity, decreases anxiety, blood pressure heart an respiratory rate and may have positive effects on sleep via muscle relaxation ad distraction from thoughts.

\section{REDUCES NEGATIVE EMOTION}

Music, especially upbeat tunes, can take mind off what stresses you and help you feel more optimistic and positive. This helps release stress and can even help you keep from getting as stressed over life's little frustrations in the future. Researchers discovered that music can decrease the amount of the cortical, a stress related hormone produced by the body in response to stress.

\section{MUSIC IMPROVE MOOD DECREASES DEPRESSION}

Music's ability to "heal the soul" is the stuff of legend in every culture bright, cheerful music is the most obvious prescription for the blues.

Music is something that every person has his or her own specific opinion about. Different people have different taste, and various types of music have many ways of leaving an impact on someone. It can be relaxing, angering, soothing, energizing and many more.

Music sends out either good or bad messages that have big impacts on how people act. People usually become friends with others who have a same taste in music as the rest of the people they hangout with, or it can be vice versa. People may not want to associate with people who have different tastes in music. 


\section{INTERNATIONAL JOURNAL Of RESEARCH -GRANTHAALAYAH \\ A knowledge Repository}

Art

People listen to the new things out there, they learn different things going on in the world and they become more open-minded because they re exposed to different people like the artists. People can use music to express themselves, in ways it can't expressed through behavior, or art. Groups of people around the world can come together and gather at concerts, shows, and venues to show what their interests and likings are and you see how many people have similar liking as you.

The popularity of music festivals shows just how much music is part of our culture, but researchers continue to find that music can also be an integral part of our health.

People can successfully improve their moods and boost their overall happiness in just two weeks. People who are going through break-ups or having relationship problems prefer music and experiences that reflect their negative mood. Music therapy has been used for centuries as a way to restore energy, improve mood, and even help the body heal more naturally. Musical time-outs as a way to calm your body and brain with soothing rhythms and to show down your heart rate and help you breathe easier.

\section{REFERENCE:-}

(1) How music affects our moods - Suzanne Boothby

(2) The power of Music to affect the brain - Elena Mannes

(3) Music and Brains - Crichtley and Henson

(4) Sangeet Visharad-Basant 